\title{
A MULTI AWARE MULTI-LEVELS HETEROGENOUS ROUTING (MAMHR) PROTOCOL FOR WIRELESS SENSOR NETWORKS
}

\author{
K.P. Indulekha, G. Jayasree and B. Malarkodi \\ Department of Electronics and Communication Engineering, National Institute of Technology, Tiruchirappalli, India
}

\begin{abstract}
Wireless Sensor Networks (WSNs) are generally set out in a remote workplace, since the sensor nodes are tiny in size, cost-proficient, lowpower gadgets, and have restricted battery supply. Due to the constrained availability of power sources, energy utilization has been considered as the most crucial factor for proposing network routing protocols. The fundamental concern is to improve the lifetime of the network based on the energy constraints. Several homogenous clusterbased routing protocols have been proposed in literature for lifetime improvement of the sensor network but many of them fail to function effectively in heterogeneous environment and moreover, these protocols have not considered any other awareness parameters than lifetime and energy consumption. In this work, a Multi Aware MultiLevels Heterogeneous Routing (MAMHR) protocol, focusing on the principle of multi-level heterogeneity by considering multiple awareness parameters of the network such as Quality of Service (QoS), shortest path estimation and suitable localization technique, is proposed. Scoped Bellman Ford Routing (SBFR) algorithm is used for shortest path estimation, Reliability, Availability, and Serviceability (RAS) factors are considered for QoS awareness and Time Difference of Arrival (TDOA) technique is used for location estimation. Lifetime awareness parameters of the proposed scheme were compared with already existing prominent protocols LEACH, SEP and ZSEP and a significant improvement in lifetime of entire network was obtained. Simulation results corresponding to the respective multiple awareness parameters also shows that these parameters can be incorporated into the selected heterogeneous environment without affecting the energy consumption constraints of the proposed scheme.
\end{abstract}

Keywords:

WSNs, Lifetime Improvement, MAMHR, QoS and TDOA Localization

\section{INTRODUCTION}

WSNs comprise of group of distinct, closely packed, and minuscule Sensor Nodes (SNs). These SNs are haphazardly deployed for the smooth handling of different applications, like military operations, precision agriculture, location dependent operations, medicine and health care operations, innovative operations, disaster relief operations, machine surveillance operations and so on. Because of the irregular distribution of claiming SNs, battery can't be renewed easily, and hence energy utilization is one of the critical problems to be considered while proposing routing protocols for sensor networks [14]. So, there exists a demand for adequate routing mechanisms that assures proper utilization of energy and extended lifetime of nodes, as well as provides an accurate estimate of the location of motes in the network. Thus, there are two desirable characteristics of a sensor network, viz. lower hardware cost, and uniform energy drainage. While heterogeneous networks achieve the former, the homogeneous networks achieve the latter [15]. However, both features cannot be incorporated in the same network.
Even though there exist many lifetime improvement routing protocols in literature, these have hardly considered other awareness parameters like Shortest path and associated cost calculation, QoS parameters of network, suitable localization technique etc [16]. The existing homogenous based clustering protocols in literature fail to perform well in heterogeneous environment. Moreover, GPS based localization estimate results in high hardware cost and large energy consumption.

Motivated by above facts, in this project, multi-level energy heterogeneity for cluster based routing protocols in WSNs has been considered for lifetime improvement of the network [17]. In addition to lifetime improvement, shortest path and associated cost calculation by assigning cost matrix which is a function of the lifetime parameters of the nodes of the associated links is also included [1]. QoS parameters of the sensor networks are evaluated in terms of Reliability, Availability, and Serviceability (RAS) for ensuring network security [18]. A suitable localization technique which is based on distributed centralized localization is selected which provides a better accurate estimation on the position estimates of nodes with less error which works on TDOA (Time Difference of Arrival) technique.

Remaining of the paper is organized as follows: Section 3 provides system description. In section 4, simulated results corresponding to lifetime improvement parameters are analyzed and compared with that of LEACH, SEP and Z-SEP protocols, Shortest path estimated is evaluated, RAS parameters are judged and TDOA method and its accuracy is cross checked. Section 5 concludes the work with proper judgments from the observations and section 6 evaluates the limitations of the work as well as foresees the scope for future improvements.

\section{LITERATURE REVIEW}

Many lifetime-extending clustering protocols has been introduced in literature. Among these routing protocols, homogenous based clustering protocols like LEACH [2] fail to perform well in heterogeneous environment, while, in vertical heterogeneous routing protocol like SEP [3] and Z-SEP [4] increasing the count of levels will not provide assurance for improvement in total initial energy of network area. Hence in this section, a study of routing protocols, optimum path finding algorithms, security integrating methods and localization techniques in literature is included.

\subsection{ROUTING PROTOCOLS AND OPTIMUM PATH FINDING ALGORITHMS IN LITERATURE}

Systems in which all the sensor nodes have similar battery vitality and equipment intricacy are called homogenous systems or networks [12]. Clustering method in such networks is stagnant 
in nature, hence the cluster head will be overburdened with communication of data to far-off destination nodes, especially when the communication range is significantly large, due to the additional computational overheads involved, since the node which is selected as cluster head has to perform this role for the whole duration of network lifespan [9]. Hence the energy drainage in cluster heads occur at a faster rate compared to normal nodes. In case of heterogeneous sensor networks, sensor nodes vary in their processing capabilities because of their variations in computing power and functionality. But implementation and topography management are more complicated in heterogeneous networks than that of homogeneous networks. The main idea is that; some selected cluster head nodes can be installed with more initial energy sources as well high complex hardware instead of incorporating in all nodes [10]. If single hop communication is used, energy consumption will be more in those nodes which are at longer distance from cluster heads. Anyhow, once the cluster head is made permanent, it is not possible to interchange the performance aspects anymore.

Bellman Ford Routing (BFR) is an algorithm that computes the shortest paths from a single source node to all of the other nodes in a weighted link network [6]. It is slower than Dijkstra's algorithm but more versatile which also supports negative weights for links. Like Dijkstra's Algorithm, Bellman-Ford is based on the principle of relaxation, in which an approximation to the correct distance is gradually replaced by more accurate values until eventually reaching the optimum solution [7]. In both algorithms, the approximate distance to each node is always an overestimate of the true distance and is replaced by the minimum of its old value and the length of a newly found path.

Based on the above facts, in this project we have opted SBFR (Scoped Bellman-Ford Routing), a robust and scalable routing protocol. The key idea is that each node maintains a view scope of the network by computing distance vectors using the distributed Bellman-Ford method [8]. Each node picks the node closest to the sink as the local forwarding target, i.e., landmark, and according to the target, it looks up the best next hop in the distance vector when forwarding a packet. Theoretical analysis shows that SBFR has a better performance in comparison with other routing protocols.

\subsection{RAS FACTORS AND QOS INTEGRATION FOR NETWORK SECURITY}

Wireless Sensor Networks being a key area of new ventures and researches that promises an advanced domain on the manner in which computer and human interact, providing accurate and appropriate information is a primary constrained for better overall performance of the network. Quality of Service (QoS) in WSN examines various approaches and fundamentals to facilitate decisive and established connection services for data transmission in the network. But, ensuring QoS in wireless sensor networks is laborious due to the mismatch in the demands to be met by the different applications and also because of the non-end-to-end communication between the nodes.

Reliability, Availability and Serviceability (RAS) are the means of which security is measured and ensured in wireless sensor networks. Reliability Reliability is the possibility that our network will function accurately for an expected time duration. Availability indicates the time extent to which the network is actually functioning out of the total duration over which it is estimated to be functioning [11]. Availability aspects permit the system to stay functional even in the presence of faults. Serviceability or maintainability is the ease with which the network can be brought back to its initial condition once it goes to fault condition. The main clashing challenge that network architects face is to reduce the total cost of the network meanwhile to furnish effective security of the network [19]. Constraints in the cost and the energy consumption will also adversely affect the reliability aspects of network. The application itself will greatly influence how system resources must be allocated between communication and computation requirements to achieve requisite system performance.

\subsection{LOCALIZATION TECHNIQUES PROPOSED IN LITERATURE}

In fields where location estimation of nodes is critical, even if Global Positioning System (GPS) can be used to determine the node location with good precision, the high hardware cost and energy requirements of GPS receivers often prohibit its ubiquitous use for location estimates [21]. Hence, there are various localization algorithms proposed in literature for low cost location estimation of nodes.

Localization is evaluated by, setting a node with known location as a reference and making it to communicate to other Non-localized nodes and hence by calculating their distance with respect to the reference nodes. In some cases, instead of geometrical relative distance, relative angle is also considered based on the technique used [20]. Localization techniques can be mainly categorized as range-free and range-based techniques. Range based schemes are distance-estimation-and angleestimation-based techniques. Important techniques used in rangebased localization are received signal strength indication (RSSI), angle of arrival (AOA), time difference of arrival (TDOA), and time of arrival (TOA). As the name suggests, in Angle of Arrival (AOA) method, angle is used for estimation, in Time of Arrival (TOA) method, time taken for arriving from a source to destination is made use of and in Time Difference of Arrival (TDOA), difference in time for a light signal and a sound signal to reach from a source to destination is used for calculating the location estimates [5]. Since the accuracy of the technique used is crucial, TDOA technique with disjoint source estimation in closed form is used in this paper.

\section{SYSTEM DESCRIPTION}

Overall description of the system including the design equations, conditions, performance parameters and initial values etc. in all the awareness cases is described in detail in this section.

\subsection{HETEROGENEOUS EPOCH CALCULATIONS AND EVALUATION OF LIFETIME METRICS}

Nodes are divided into multiple levels called normal, first level advanced, second level advanced, third level advanced and $k$ level advanced nodes based on the value of initial residual energy values. Matching with other antecedent cluster-based routing protocols in literature [2], MAMHR was performed in two stages as: setup and steady state. Division of nodes into k different 
levels of energy heterogeneity in horizontal manner is shown in Fig.1.

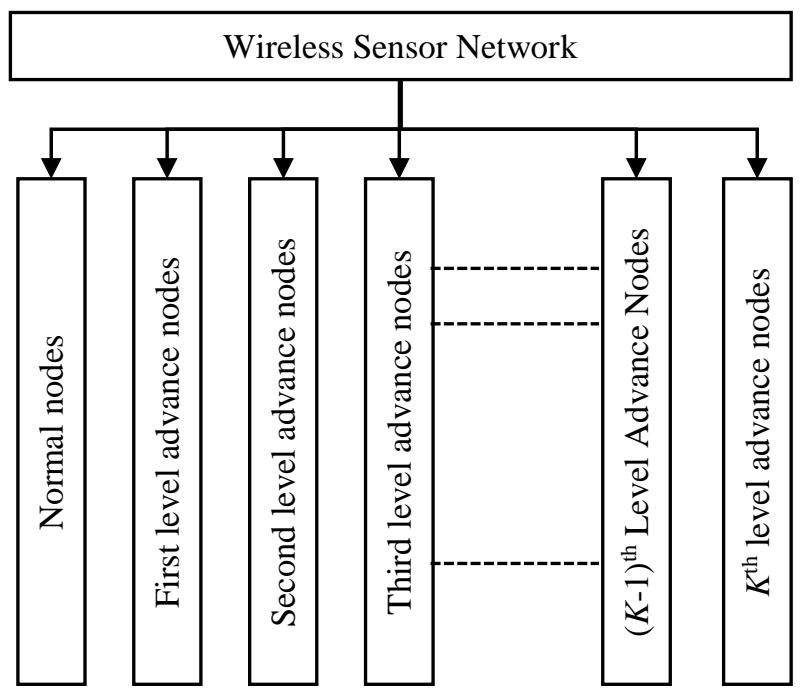

Fig.1. K- Level Horizontal Energy Heterogeneity

For upturning the accuracy of system, heterogeneous epoch has to be equal to $\frac{1+\sum_{i=1}^{k} a_{i} * m_{i}}{P_{o p t}}$. Average number of Cluster Heads per round corresponding to each epoch is made steady and equal to $N * p_{\text {opt }}$ for legitimate stabilizing of energy. Hence, probabilities of election for the first, second,..., $(k-1)^{\text {th }}$ and $k^{\text {th }}$ levels of sensor nodes are as follows:

$$
\begin{gathered}
P_{n}=\frac{P_{o p t}}{1+\sum_{i=1}^{k} m_{i} * a_{i}} \\
P_{a 1}=\frac{P_{o p t}\left(1+a_{1}\right)}{1+\sum_{i=1}^{k} m_{i} * a_{i}} \\
P_{a 2}=\frac{P_{o p t}\left(1+a_{2}\right)}{1+\sum_{i=1}^{k} m_{i} * a_{i}} \\
P_{a_{k-1}}=\frac{P_{o p t}\left(1+a_{k-1}\right)}{1+\sum_{i=1}^{k} m_{i} * a_{i}} \\
P_{a k}=\frac{P_{o p t}\left(1+a_{k}\right)}{1+\sum_{i=1}^{k} m_{i} * a_{i}}
\end{gathered}
$$

After calculating the probability of election of nodes at different levels, threshold level for Cluster Head selection can be calculated using the following equations:

$$
T\left(S_{n}\right)=\left\{\begin{array}{cc}
\frac{P_{n}}{1-P_{n}\left(r \bmod \frac{1}{P_{n}}\right)} & \text { if } S_{n} \in G \\
0 & \text { otherwise }
\end{array}\right.
$$

where, $r$ represents on-going round, $G$ stands for the group of normal nodes that have not turned into Cluster Heads within last $1 / P_{n}$ rounds, and $T\left(S_{n}\right)$ indicates the threshold corresponding to a group of $N * \sum_{i=1}^{k} m_{i}$ normal nodes.

$$
T\left(S_{a_{1}}\right)=\left\{\begin{array}{cc}
\frac{P_{a_{1}}}{1-P_{a_{1}}\left(r \bmod \frac{1}{P_{a_{1}}}\right)} & \text { if } S_{a_{1}} \in G_{1} \\
0 & \text { otherwise }
\end{array}\right.
$$

where, $G_{1}$ stands for a group of first level nodes which have not turned into $\mathrm{CHs}$ for the last $1 / P_{a_{1}}$ rounds and $T\left(S_{a_{1}}\right)$ indicates threshold for a set of $N^{*} m_{1}$ level one advanced nodes.

$$
T\left(S_{a_{k}}\right)=\left\{\begin{array}{cc}
\frac{P_{a_{1}}}{1-P_{a_{k}}\left(r \bmod \frac{1}{P_{a_{k}}}\right)} & \text { if } S_{a_{k}} \in G_{k} \\
0 & \text { otherwise }
\end{array}\right.
$$

where $G_{k}$ stands for a group of $k^{\text {th }}$ level nodes that have not become CHs within last $1 / P_{a_{k}}$ rounds, and $T\left(S_{a_{k}}\right)$ indicates the threshold corresponding to a group of $N^{*} m_{k}$ advanced nodes in level $k$. As a result, increase in count of horizontal energy levels will ensure a significant increase in total initial energy of system field as well as count of nodes in advanced level will not depend on preceding level.

Lifetime parameters adopted in this paper are:

- Count of alive nodes in each round.

- Count of dead nodes in each round.

- Throughput, i.e. the count of packets sent to base station.

At starting all the nodes are assumed to have some residual initial energy. As the time progresses, the energy gets exhausted and the energy of individual nodes fails to meet the minimum required criteria. The nodes whose energy levels falls below the minimum threshold nodes are taken as dead nodes and those nodes which still have remaining energy in them are considered as alive nodes. After running protocols for 9000 rounds, the number of dead nodes, alive nodes and throughput of the network were plotted for LEACH, SEP, Z-SEP and HMHR for comparison of lifetime.

Table.1. Heterogeneity Parameters Used for Simulation

\begin{tabular}{|c|c|c|c|c|c|}
\hline \multicolumn{6}{|c|}{ Heterogeneity Parameters } \\
\hline$m_{1}$ & $m_{2}$ & $m_{3}$ & $a_{1}$ & $a_{2}$ & $a_{3}$ \\
\hline 0.4 & 0.3 & 0.1 & 1 & 2 & 3 \\
\hline
\end{tabular}

In this paper, a network subsisting of 100 nodes, deployed randomly in a $100 \times 100$ area and a base station located in the centre is considered in MATLAB platform. LEACH, SEP and ZSEP and proposed horizontal based protocol are set up with equal number of nodes, taking the same standard radio energy model as in [2] with fixed initial energy $E_{o}$ as $0.5 \mathrm{~J}$. For simulation purpose, the value of $k$ selected was 3 and hence the nodes were classified 
into normal nodes, level 1 advanced nodes, level 2 advanced nodes and level 3 advanced nodes.

Simulations corresponding to the heterogeneity parameter values as per the Table. 1 were performed in MATLAB using Eq.(1) to Eq.(5) for calculating probability, and Eq.(6) to Eq.(8) for cluster head selection respectively. Keeping the initial energy as well as other parameters of the sensor network environment as constant, the model is simulated in MATLAB and compared with other three main prominent pre-existing protocols, LEACH, SEP and Z-SEP.Data transmission from a source was performed after selecting the suitable cluster heads and by calculating the distance to cluster heads and sink location. Cluster head distribution of the network is done based on the values of heterogeneity parameters in such a way that there is an available associated cluster head for each node in the network, for an effective data transmission to the sink node.

\subsection{SHORTEST ESTIMATION}

TRANSMISSION

PATH

For finding optimum path from source to destination, we are using SBFR, which is a combination of Bellman-Ford routing and cost-based geographic routing. Bellman-Ford routing is an adequate method to achieve route optimality and geographic routing is a potential way to accomplish improved scalability. In SBFR, each node maintains an $H$-hop view scope of the network by computing distance vectors using the distributed Bellman-Ford method. A cost vector named estDist is maintained and refreshed based on values of lifetime metrics. A node chooses the node in its view scope with least estDist as a temporary landmark for forwarding a packet. According to the landmark node, it checks for the best next hop in the routing table. SBFR uses the costbased approach for reducing the void problem and selects the node with least cost within the scope as the landmark.

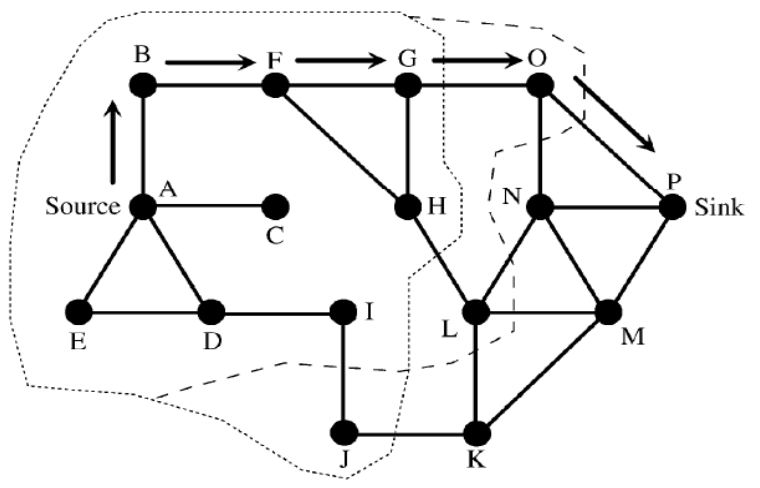

Fig.2. Landmark Guided Forwarding

Parameters in the routing table corresponding to a node $u$ can be summarizes as:

$$
\begin{gathered}
R T u=f(\text { destID } ; \text { destPos; estDist } ; \text { estVoids; hops;nextHop; } \\
\text { entryStatus; } \text { entryChanged; seqNum })
\end{gathered}
$$

where, destID is the identification of a destination node; destPos is the respective location of that destination; estDistis the evaluated transmission distance from that node to the sink; estVoids is the calculated number of voids that node destIDis is associated with; hops is the minimum number of hops from node $\mathrm{u}$ to that node; nextHop is the next hop on the optimum path from node u to that node; entryStatus represents the conditional status of that entry, entryChanged indicates whether the entry is modified or not; seqNum is the sequence number depicting timeliness.

Table.2. Routing Table of Node $A$

\begin{tabular}{|c|c|c|c|}
\hline destID & estDist & Hops & nextHop \\
\hline A & 4.000 & 0 & A \\
\hline B & 4.123 & 1 & B \\
\hline C & 3.000 & 1 & C \\
\hline D & 3.606 & 1 & D \\
\hline E & 4.583 & 1 & E \\
\hline F & 3.162 & 2 & B \\
\hline G & 2.236 & 3 & B \\
\hline H & 2.000 & 3 & B \\
\hline I & 2.646 & 2 & D \\
\hline J & 3.120 & 3 & D \\
\hline
\end{tabular}

The Fig. 2 indicates the landmark guided forwarding approach used in the SBFR protocol. Let us assume $P$ as the destination node and $A$ has a packet to dispatch. Each node preserves a 3-hop view scope. The Table. 2 shows the routing table corresponding to node $\mathrm{A}$ at a partial stage. If we consider the view scope of $A$, node $H$ is the landmark for forwarding with a minimum estDist value of 2.000. Hence node $A$ passes the packet to node $B$ which is the next hop on the optimum feasible path to $H$ in the visibility range of A. Node $\mathrm{O}$ acts as the landmark for node $B$ with the least value for estDist as 1.414 which is clearly depicted Table.3, which represents the partial routing table of node $B$. Directed by node $\mathrm{O}$, the packet reaches node $F$ where the sink node $P$ can be seen.

Table.3. Routing Table of Node $B$

\begin{tabular}{|c|c|c|c|}
\hline destID & estDist & Hops & nextHop \\
\hline B & 4.123 & 0 & B \\
\hline A & 4.000 & 1 & A \\
\hline C & 3.000 & 2 & A \\
\hline D & 3.606 & 2 & A \\
\hline E & 4.583 & 2 & A \\
\hline F & 3.162 & 1 & F \\
\hline G & 2.236 & 2 & F \\
\hline H & 2.000 & 2 & F \\
\hline I & 2.646 & 3 & A \\
\hline L & 1.732 & 3 & F \\
\hline O & 1.414 & 3 & F \\
\hline
\end{tabular}

The logic used in SBFR for establishing routing table is quite similar to DSDV. Routing table $R T u$ corresponding to each node $\mathrm{u}$ is initialized with only the entry associated with itself at starting. For calculating the estDist, sink broadcasts its position to the whole network and each node can find its relative position with respect to sink. After initialization, each node may be prompted to advertise its routing table to its neighbours. When a node obtains a neighbour's routing table, say $R T$ neigh, it modifies its own as per the information received from that routing table. 
The method used in SBFR to trigger updates of routing tables is as follows.

Whenever there is a variation in the location of node $u$, it modifies $R T u$ by updating the dest $P$ and computing its estDist afresh. Practically considering minimization of updating cost as well, an updating is incorporated if and only if the change in position is greater than a predefined value which is taken as 3 meters here in simulations.

Every time the routing table changes, node $u$ refreshes the very entry whose destID is equal to $u$ by once again calculating the estDist. If any change is reflected in its estDist field, then node $u$ urgently advertises the change of its routing table to its neighbours. As soon as the routing table changes, node $u$ updates the very entry whose destID is equal to $u$ by computing estDist once more. It only broadcasts those changed entries whose hops is less than or equal to $(H ; 1)$, where $H$ is the view scope in terms of number of hops.

No matter whether the routing table changes or not, each node (except those which are unaware of sink position and those supposed to be disconnected) periodically broadcasts to its neighbours all the entries whose hops is less than or equal to ( $H$ i 1$)$.

For finding whether a node has failed or dead, a lifetime duration boundary value is set (in simulation it is fixed as 60 seconds). If any of the entries that has not been updated for duration more than this boundary value, then that corresponding node is assumed to be dead or failed and hence entry is removed.

Each node in the network, updates its own routing table soon after collecting the updating information from the neighbour's routing table.

\subsection{SECURITY INTEGRATION AND RAS CALCULATION}

For ensuring the security in WSNs, reliability, availability and serviceability are considered as the parameters of QoS evaluations. Reliability of a system is defined as the probability of system survival in a given duration of time. Mean Time Between Failure $(M T B F)$ is used for calculation of reliability since it is highly influenced by the time of operation as well as condition of operating environment. If $t$ denotes the time duration, reliability as a function of MTBF can be expressed as:

$$
\text { Reliability }=1-(t / M T B F)
$$

Probability of a system to perform accurately at a given time period is called its availability. Given the $M T B F$ and Mean Time To Repair (MTTR), availability can be calculated using:

$$
\text { Availability }=M T B F /(M T B F+M T T R)
$$

Hence, for the betterment of performance in terms of availability, MTTR of the system has to be minimum as well as $M T B F$ has to be maximum. $M T B F$ is dependent on the fabrication model and hence difficult to control, but MTTR can be restricted in accordance with the node deployment and network composition.

Serviceability of a system is determined by the probability that the system will be able to recover back to its initial condition of operation after a breakdown. It can be calculated as:

$$
\text { Serviceability }=1-e^{-\frac{t}{M T T R}}
$$

In case of sensor networks, breakdown rate or failure rate can be evaluated by considering the number of dead nodes and the corresponding number of rounds of execution.

$M T B F$ in sensor nodes is measured by using the characteristic breakdown rates for each element separately. Assuming the breakdown rates $(\lambda)$ of individual redundant units as same, $M T B F$ of overall system with $\mathrm{n}$ lateral autonomous units can be taken as:

$$
M T B F=\sum_{i=1}^{N} \frac{1}{i \lambda}
$$

The MTTR can be obtained by the addition of Mean Time to Detect $(M T T D)$ the failures and the Time to Repair (TTR) (MTTR $=M T T D+T T R)$ which can be highly influenced by the network conditions.

If a breakdown occurs, the consumer will send the repair message to the sensor node and load the backup component. Acknowledgement is sent back to the consumer after finishing the reparation. If the message latency from the consumer to the target node is d seconds and the test time is c seconds, then we calculate MTTR as per Eq.(13).

$$
M T T R \sim 4 d+c
$$

The destination node transmits the consistent data request to the failed node. Same request is sent to neighbouring nodes within a predefined proximity to measure the truthfulness of the data by aggregating these data packets, it is possible to figure out the faulty node. After detecting failure, a command is send to the neighbouring nodes to indicate about the faulty node. If $d$ denotes the delay involved in transmitting message from the consumer node to the destination node, then MTTR is given by,

$$
M T T R \sim 8 d
$$

Reliability of a system is defined as the probability of system survival in a period of time. Therefore, using Poisson probability implemented for WSNs we have as well estimate probability of "failed" situation for whole WSN in given time interval

$$
\operatorname{probability}(r)=\frac{m^{r} * e^{-m}}{r !}
$$

where probability $(r)$ is a probability of failure system working with $r$ failed nodes within WSN for given time interval is an average number of failed nodes within WSN.

\subsection{TDOA LOCALIZATION AND ACCURACY MEASUREMENTS}

Procedure for the TDOA localization technique used in this paper and corresponding accuracy measurement can be summarized as follows:

Let there be $\mathrm{N}$ sources with locations $U_{i}=\left[X_{i} Y_{i} Z_{i}\right], i=1$, $2, \ldots, N$, are unknown and has to be located. Let $S_{j}=\left[X_{j} Y_{j} Z_{j}\right] j=$ $1,2, \ldots, M$ denotes position corresponding to the receiver sensors that detects the signals sent from respective sources. The TDOAs of the received signals with respect to a reference sensor are estimated. Here disjoint sources are selected for getting distinct TDOA values corresponding to each sending sources. For this, while measuring the TDOA responses, signals from the sources are constrained to disjoint intervals of time. 
Location of sensors accessible for processing, $S_{j}$, tends to have arbitrary errors. $S_{j}$ can be assembled to obtain sensor location vector.

$$
\begin{gathered}
S=\left[S_{1}^{T}, S_{2}^{T}, \ldots, S_{M}^{T}\right]^{T} \\
\nabla S=S-S^{0}
\end{gathered}
$$

Here, $\quad \nabla S=\left[\nabla S_{1}^{T}, \nabla S_{2}^{T}, \ldots, \nabla S_{M}^{T}\right]^{T}$, where $\quad \nabla S_{j} \quad$ denotes location errors present in $S_{j}$, and $S^{0}=\left[S_{1}^{0 T}, S_{2}^{0 T}, \ldots, S_{M}^{0 T}\right]^{T}$.

$\nabla S$ is considered as a Gaussian random vector with zero mean whose covariance matrix is given by $Q_{b}$. Hence, obtained TDOA estimates are expected approaching the true sensor positions $S^{0}$ in time. Let $d_{j 1, i}$ indicates the TDOA estimate among sensor pair $j$ and 1 from the $i^{\text {th }}$ source position, where $j=1,2, \ldots, M$ sensor 1 being the reference sensor for the estimation.

$\nabla S$ is considered as a Gaussian random vector with zero mean whose covariance matrix is given by $\mathrm{Qb}$. Hence, obtained TDOA estimates are expected approaching the true sensor positions $S^{0}$ in time. Let $d_{j 1, i}$ indicates the TDOA estimate among sensor pair $j$ and 1 from the $i^{\text {th }}$ source position, where $j=1,2, \ldots M$, sensor 1 being the reference sensor for the estimation.

Gathering the TDOAs from the same origin in a wellorganised manner and multiplying by the propagation speed $c$ of the signal to form $N$ range difference-of-arrival (RDOA) vectors as follows:

$$
\begin{gathered}
r_{i}=\left[r_{21, i}, r_{31, i}, \ldots, r_{M 1, i}\right]^{T} \\
r_{i}=c \cdot\left[d_{21, i}, d_{31, i}, \ldots, d_{M 1, i}\right]^{T} \\
r_{i}=r_{1}^{0}+n_{i}
\end{gathered}
$$

Here, $\quad r_{1}^{0}=\left[r_{21, i}^{0}, r_{31, i}^{0}, \ldots, r_{M 1, i}^{0}\right]^{T}, n_{i}=\left[n_{21, i}, n_{31, i}, \ldots, n_{M 1, i}\right]^{T}$ and $n_{J 1, i}$ is the noise in RDOA which is $c$ times the noise present in $d_{J 1, i}$. Let actual distance between the source $u_{i}^{0}$ and sensor $j$ be $r_{j, i}^{0}$, i.e.,

$$
r_{j, i}^{0}=\left\|u_{i}^{0}-s_{j}^{0}\right\|
$$

Here, $\|*\|$ stands for Euclidean norm. Hence, the actual RDOA $r_{j 1, i}^{0}$ is:

$$
r_{j 1, i}^{0}=r_{j, i}^{0}-r_{1, i}^{0}
$$

Assemble all RDOA estimates together to form the RDOA vector $r=r^{0}+n$.

The estimate vector corresponding to noise $n=\left[n_{1}^{T}, n_{2}^{T}, \ldots, n_{N}^{T}\right]^{T}$ is assumed as a Gaussian random vector with zero mean and covariance matrix $Q_{b}$. This Gaussian hypothesis can be supported from the TDOA evaluation procedure. In most instances, the estimate among a sensor pair is acquired by the way of finding the peak position in the derived cross-correlation of the obtained signals.

In this paper, the localization technique for two disjoint sources is considered. This algorithm which is adopted for the two-source scenario can be extended to the scenario with random number of radiating origins. For validating the accuracy of estimation, Mean Square Error plot is compared with standard Cramer Rao Lower Bound (CRLB) plot. Mean Square Error is evaluated as:

$$
\operatorname{MSE}(u)=\frac{\sum_{i=1}^{L}\left\|u^{i}-u^{0}\right\|^{2}}{L}
$$

where $L$ represents the number of ensemble runs.

The noisy sensor position covariance matrix is noted as $Q_{b}=\sigma_{s}^{2} J$ and true RDOA measurement covariance matrix as $Q_{a}=\sigma_{r}^{2} J$, respectively. Then $Q_{b} / Q_{a}$ vs MSE curves corresponding to both the sources are plotted and evaluated to judge the accuracy of the localization technique by plotting in logarithmic scale.

\section{SIMULATION RESULTS}

Simulation results obtained for the selected scenario of wireless network with 100 nodes for different awareness parameters, corresponding analysis and observations are included in this section.

\subsection{COMPARISON WITH LEACH, SEP AND Z-SEP IN TERMS OF LIFETIME IMPROVEMENT}

The number of alive nodes initially is set equal to the total number of nodes deployed in the given area. In each round of MATLAB simulation, energy of the individual nodes is calculated and updated.

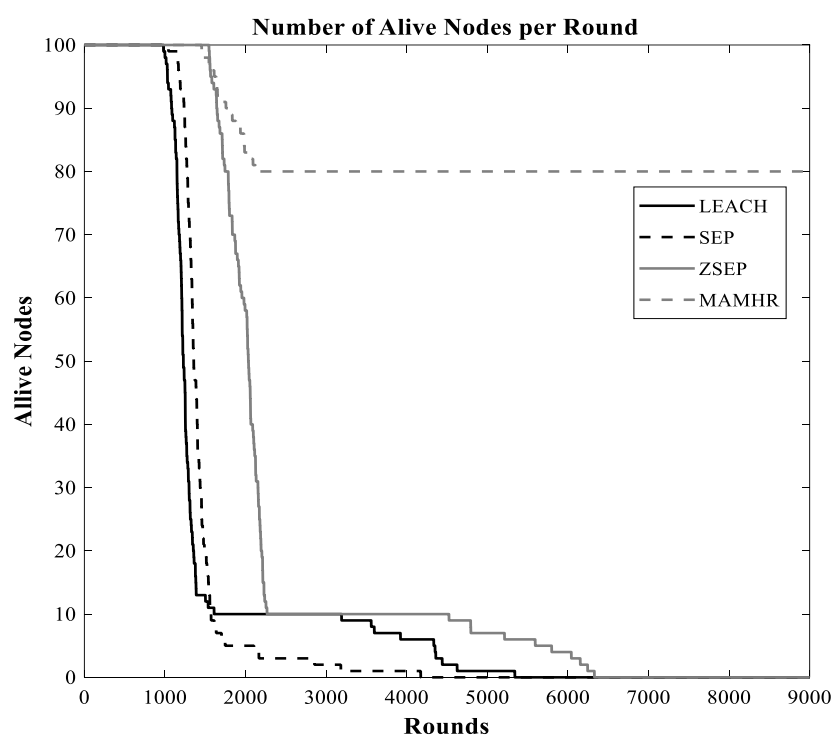

Fig.3. Comparison between number of Alive Nodes

Hence as time progresses, energy of individual nodes decreases gradually which results in decrease in number of alive nodes in the network at a given time as shown in the simulated Fig.3. The count of alive nodes in LEACH and SEP are almost same, and Z-SEP shows better performance compared to these two since network is divided into three zones in it [4]. It is clearly seen in this Fig. 4 that there is a significant improvement in the alive nodes plot in case of MAMHR compared to all other protocols due to its horizontal multi-level distribution. Initially 
there are no dead nodes present in the network since all nodes are provided with a residual amount of initial energy.

As per the simulation result shown in Fig.4 in LEACH, SEP and Z-SEP almost all the nodes die by around the first 5000 rounds, while in MAMHR number of dead nodes is in the range of 20 for the evaluated 9000 rounds, which clearly indicates the improvement in lifetime of the proposed MAMHR.

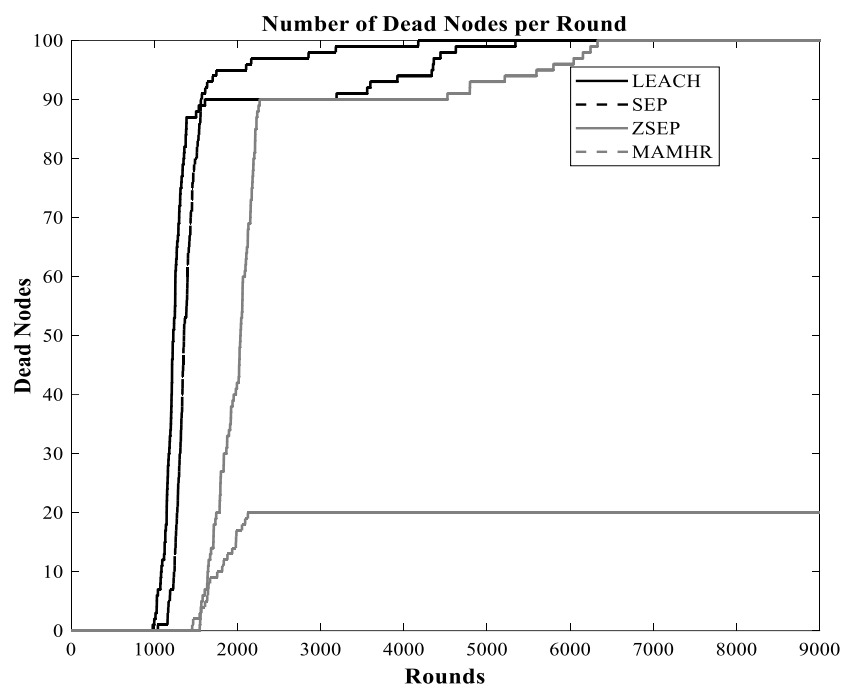

Fig.4. Comparison between number of Dead Nodes

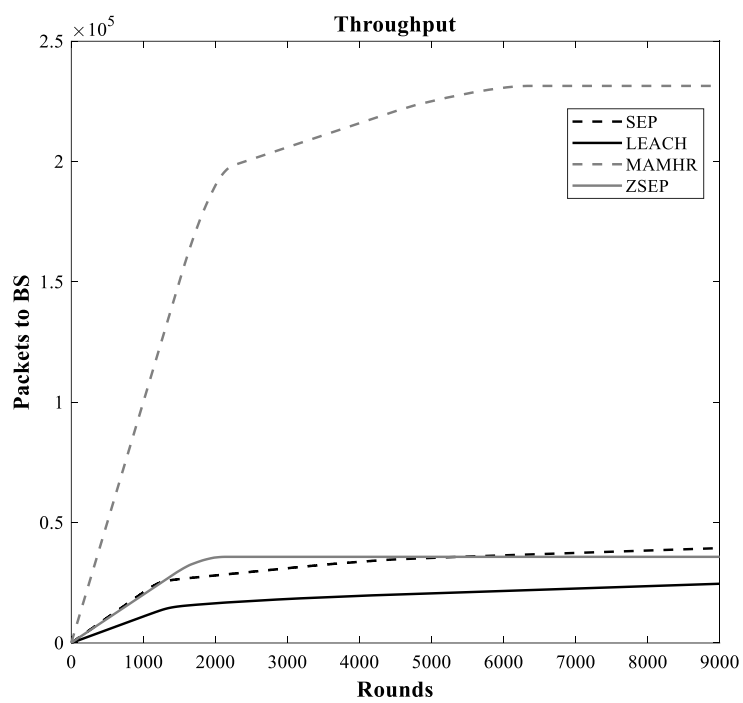

Fig.5. Comparison between throughput of the Network

The Fig. 5 shows the contrast among the protocols considering the packets obtained at the base station with increment in number of rounds. The rate increases with the increment in residual energy and stability of the corresponding protocol increases accordingly. However, MAMHR throughput curve appears a long way better than curves corresponding to protocols SEP, Z-SEP and LEACH. Overall performance of SEP and Z-SEP are nearly similar. Among SEP and LEACH, performance level of SEP is higher than LEACH.

As in LEACH [2], there may be an identical chance of every node to emerge as cluster head. Hence, chances are there for the advanced nodes to emerge as cluster head again in next round, and the normal nodes lack capability to perform better in case of packet aggregation and communication when they get elected as cluster head. It decrements the count of packets transferred to the base station. At the same time as in SEP [3], there is a system for weighted election probability for advanced and normal nodes. While in MAMHR, weighted election probability which ensures horizontal level distribution and increase in energy of nodes with increase in number of levels, makes its performance the best compared to other protocols.

\subsection{SHORTEST PATH ESTIMATION AND COST CALCULATION}

Based on the value of heterogeneity parameters, shortest path estimation method is selected.

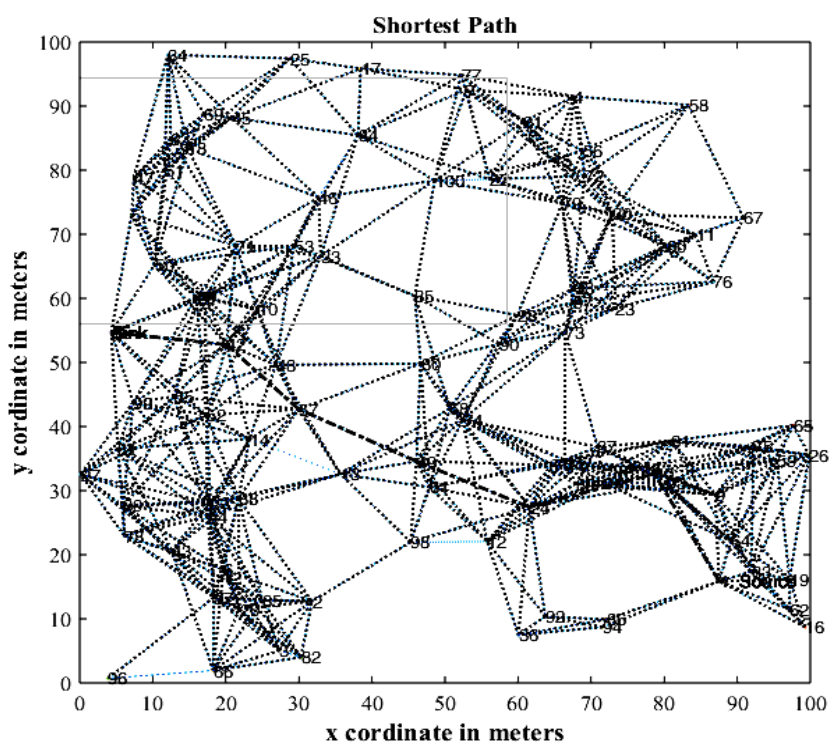

Fig.6. Shortest Path Calculation in Homogenous Environment

Cost of a link is considered as a function of the lifetime of the nodes associated with that link, number of cluster heads associated, delay time of data transmission etc. If the heterogeneity parameter values are selected in such a way that the level 1 and level 2 nodes are distributed with all having same energy values, cost of all links are assumed to be same and hence the simple bellman ford algorithm with unity value as cost for links is used for shortest path estimation. In that case cost obtained is same as that of the hop count from a source to a destination.

Table.4. Shortest Path with Uniform Cost Assignment

\begin{tabular}{|c|c|}
\hline Parameter & Value \\
\hline Source ID & 1 \\
\hline Destination ID & 95 \\
\hline Cost & 6 \\
\hline Path & $1-40-24-49-57-42-95$ \\
\hline
\end{tabular}

The Table. 4 shows the shortest path available from node 1 to node 95 in terms of hop count when evaluated in homogenous environment. 


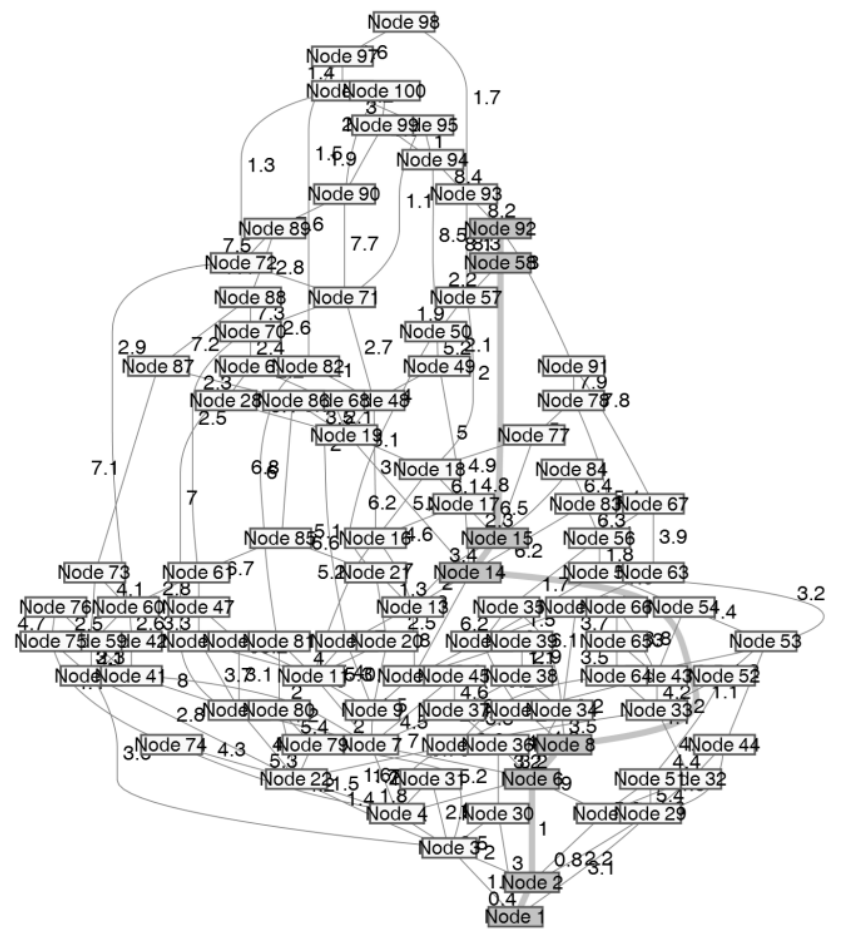

Fig.7. Shortest Path Estimation Using SBFR for 100 Nodes Deployment

On the other hand, if the heterogeneity parameters are selected in such a way that there is significant difference between the energy of nodes in each level, cost matrix is calculated based on the lifetime of the nodes associated with that link, number of cluster heads associated and delay time of data transmission and assigned to the links. Number of dead nodes, number of alive nodes, remaining residual energy of nodes, selected cluster head coordinates etc. are considered for cost matrix calculation. If the number of nodes is less than 50, shortest path estimation technique like simple bellman ford can be used for reducing hardware complexity.

Since in this scenario, number of nodes is significantly high, SBFR is used in an area of 100 node deployment, for better accuracy irrespective of hardware cost. The shortest path from node 1 to node 95 using SBFR Protocol estimated with the help of graphical method in MATLAB shown in Fig.7.

The respective cost estimate is shown in Table.5. Here the cost for reaching from the same source 1 to same destination has increased to 18.7 , cost values of individual nodes being assigned on a scale of 1 to 10 . Here, these cost values assigned are functions of the lifetime parameters of the network and energy of the individual nodes and associated cluster heads. Hence when heterogeneous parameters are too high, hardware cost of data transmission in the proposed scheme has significantly increased.

Table.5. Shortest path with non-uniform cost assignment

\begin{tabular}{|c|c|}
\hline Parameter & Value \\
\hline Source ID & 1 \\
\hline Destination ID & 95 \\
\hline Cost & 18.7 \\
\hline Path & $1-2-6-7-41-59-60-72-96-95$ \\
\hline
\end{tabular}

From these two simulations, it can be observed that there must be a tradeoff between the associated hardware cost and the lifetime improvement. Even though, there is a significant improvement in lifetime of the network around 50\% in MAMHR that utilizes $k$ level heterogeneity of nodes, hardware cost increases significantly as heterogeneity increases. So, for optimum working, parameters are evaluated first, and if they are close to homogenous values, simple bellman ford technique with unit cost assignment to individual links and when heterogeneous parameters are too high, separate cost assignment for the links based on the network parameters respectively can be used.

\subsection{EVALUATION OF QOS PARAMETERS OF THE NETWORK}

To ensure the security of the network, RAS factors corresponding to QoS of network were plotted. Security of WSN is considered through QoS. Using QoS components, we evaluated models and system-level test using sensor nodes. RAS factors which measures the QoS of the network, Reliability, Availability and Serviceability, each was evaluated separately and plotted in case of the proposed heterogeneous network of 100 nodes in order to judge to how much extent it is possible to improve the lifetime parameters without effecting performance quality.

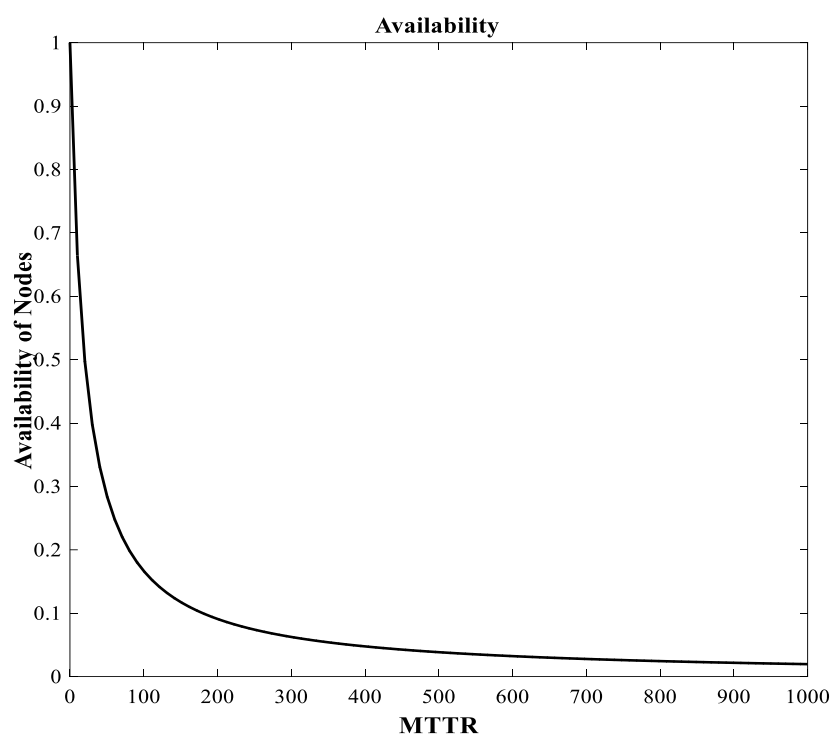

Fig.8. Availability vs MTTR Curve of the Network 


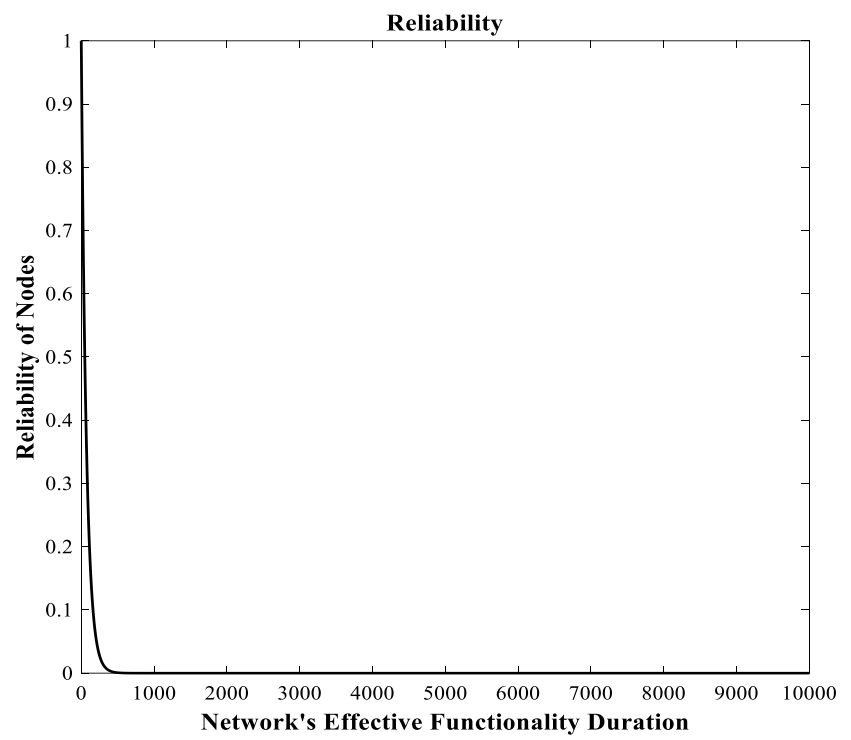

Fig.9. Reliability Curve for the Network

The Fig. 8 shows the availability plot vs MTTR in hours. MTTR values are estimated based on the lifetime parameters obtained from the simulation results. Hence it is observed that with the proposed lifetime parameters, availability of the wireless sensor network is functioning within the expected limits.

The Fig.9 shows the reliability plot of the network. Time in hours for which the network functions effectively is plotted on $x$ axis and the corresponding value of reliability using equation is plotted on $y$ axis. Since reliability curve is satisfying the required value for around 1000 hours, integrity of data transmitted is conserved since reliability reflects the data integrity.

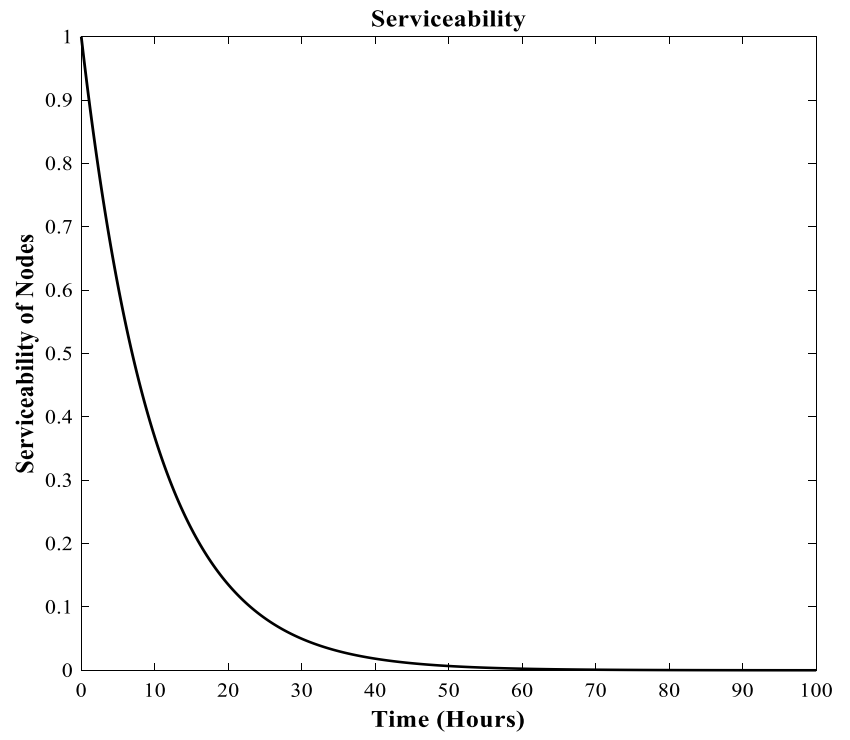

Fig.10. Serviceability Curve for the Network

The Fig.10 shows the serviceability plot for the proposed sensor network. Since serviceability indicates the probability that a failed system will restore to the correct operation, it was calculated considering repair rate and the MTTR using Eq.(11). Serviceability values within the time bound are found to match with the proposed energy consumption bound of the network for lifetime improvement.

\subsection{TDOA LOCALIZATION TECHNIQUE AND ACCURACY EVALUATION}

TDOA based estimated locations is shown in Fig.11. $N$ value is selected as 2 since two sources are used for disjoint estimation of the sensor node positions. True location of nodes estimated location of node, and location of disjoint sources used are shown. Position estimates of the sensor nodes are found using the Eq.(16) - Eq.(19). It can be observed that even if there's a slight difference from the actual sensor node positions, estimated locations lie within the region of maximum possible error zone for accurate localization of motes.

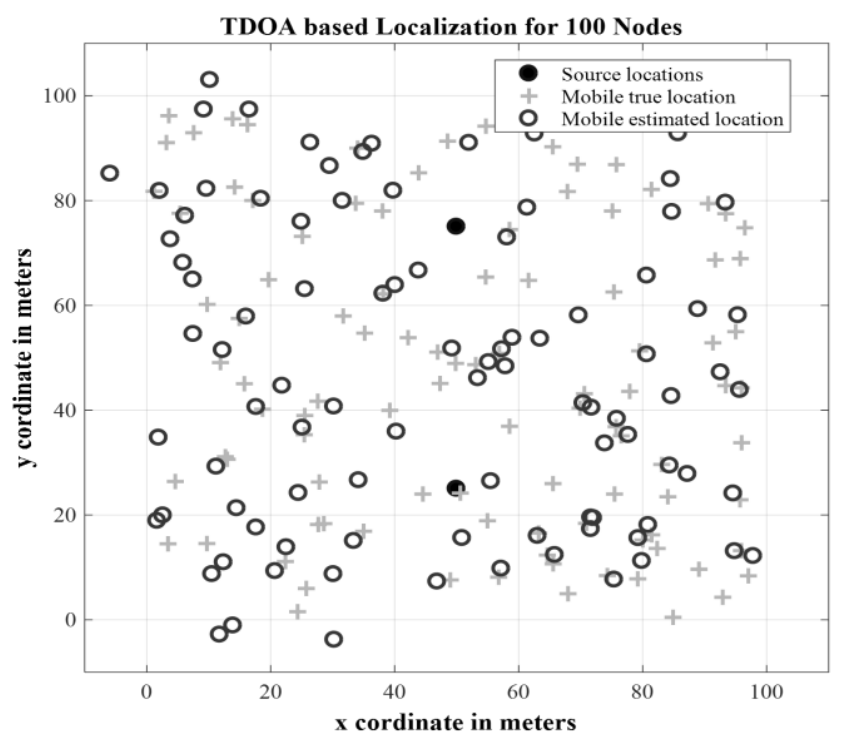

Fig.11. Location Estimates using TDOA Technique

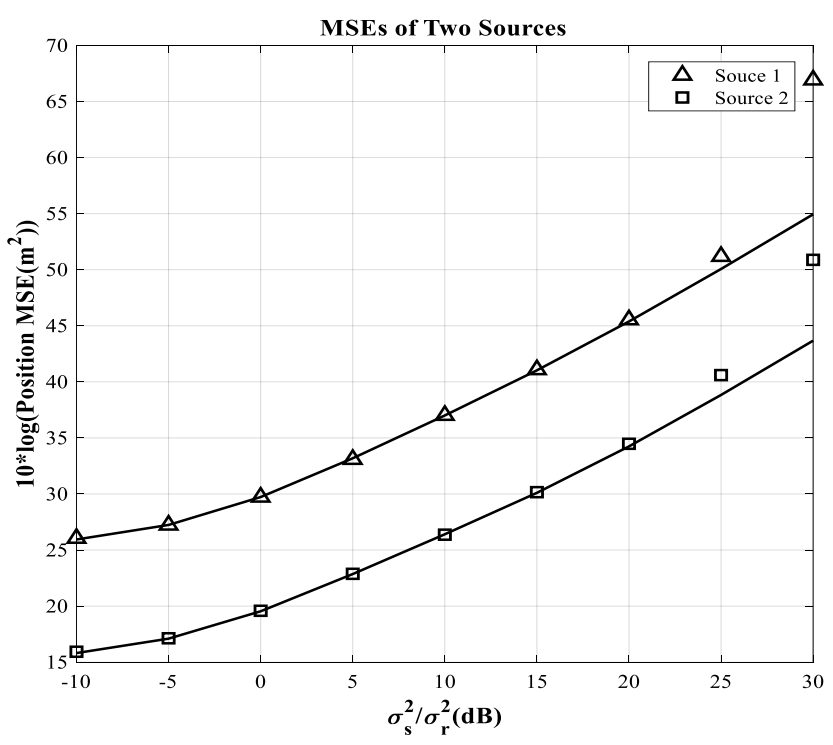

Fig.12. MSE curve approaching CRLB Limit

MSE curve obtained for the localization technique is shown in Fig.12. Ratio between noisy sensor position covariance and true RDOA measurement variance, $Q_{b} / Q_{a}\left(\sigma_{s}^{2} / \sigma_{r}^{2}\right)$ in $\mathrm{dB}$ is plotted along $x$ axis and the corresponding MSE value calculated using Eq.(20) in logarithmic scale is plotted along $y$ axis. Solid lines 
indicate the theoretical CRLB plot and the symbols indicate respective simulated result of the two sources. It can be observed that for lower values of the variance ratios for around $30 \mathrm{~dB}$, both the simulated results are approaching the CRLB limits.

\section{CONCLUSIONS}

This thesis presents a Multi Aware Multi levels Heterogeneous Routing (MAMHR) protocol, which mainly focuses on the lifetime improvement of the wireless sensor network by adopting a horizontal heterogeneous clustering method. In addition to lifetime improvement, this protocol considers other awareness parameters also which includes an effective shortest path and corresponding cost calculating algorithm, validating QoS parameters of the proposed network and a TDOA localization technique in closed form which estimates location of sensor nodes in required scenarios. Lifetime deciding parameters were simulated and compared with prominent pre-exiting protocols LEACH, SEP and ZSEP and a significant improvement in lifetime of around $48 \%$ was observed. Shortest path from a given source to destination and corresponding cost was found in an optimal way by considering the link cost as functions of heterogeneous lifetime parameters. QoS factors RAS of the system was evaluated in the proposed environment and plots were observed to be within the desired limits that ensures security of the network. Using TDOA localization technique, estimated mote locations and the MSE error curves were found to be approaching the CRLB bound which justifies the accuracy of the technique.

\section{LIMITATIONS AND SCOPE FOR FUTURE WORK}

Size of the network in terms of maximum number of nodes and hardware complexity of implementation are the major limitations of the proposed scheme. As the number of nodes increases, MSE value in localization increases which indicates reduction in accuracy of localization estimate and the cost of routing estimated using SBFA also increases which is a measure of hardware complexity of proposed method. Since it was developed in MATLAB platform, there's a limitation in evaluating with more number of nodes as well as imparting mobility features to the nodes. For betterment of the scheme evaluation and development of large and mobile network, network simulation tools like OPNET 14.5 and higher versions can be used by incorporating fuzzy interference systems that select routing based on selected awareness parameters.

\section{REFERENCES}

[1] Sudhanshu Tyagi, Sudeep Tanwar, Sumit Kumar Gupta, Neeraj Kumar and C. Rodrigues, "A Lifetime Extended Multi-Levels Heterogeneous Routing Protocol for Wireless Sensor Networks", Telecommunication Systems, Vol. 59, No. 1, pp. 43-62, 2015.

[2] Rajesh Patel, Sunil Pariyani and Vijay Ukani, "Energy and Throughput Analysis of Hierarchical Routing Protocol(LEACH) for Wireless Sensor Networks",
International Journal of Computer Applications, Vol. 20, No. 4, pp. 32-36, 2011.

[3] G. Smaragdakis, I. Matta and A. Bestavros, "SEP: A Stable Election Protocol for Clustered Heterogeneous Wireless Sensor Networks", Master Thesis, Department of Computer Science, Boston University, 2004.

[4] Banoth Thirumala, B. Vijay Kumar and Aloth Sowjanya, "Zonal-Stable Election Protocol for Wireless Sensor Networks", International Journal of Ethics in Engineering and Management Education, Vol. 3, No. 10, pp. 23-29, 2016

[5] G. Han, H. Xu, T.Q. Duong, J. Jiang and T. Hara, "Localization Algorithms of Wireless Sensor Networks: A Survey", Telecommunication Systems, Vol. 52, No. 4, pp. 2419-2436, 2013.

[6] R. Kozick and B. Sadler, "Source Localization with Distributed Sensor Arrays and Partial Spatial Coherence", IEEE Transactions on Signal Processing, Vol. 52, No. 3, pp. 601-616, 2004.

[7] K.C. Ho, X. Lu, and L. Kovavisaruch, "Source Localization using TDOA and FDOA Measurements in the Presence of Receiver Location Errors: ANALYSIS and Solution", IEEE Transactions on Signal Processing, Vol. 55, No. 2, pp. 684696, 2007.

[8] A. Perrig, J. Stankovic and D. Wagner, "Security in Wireless Sensor Networks", Communications of the ACM, Vol. 47, No. 6, pp. 53-57, 2004.

[9] M.W. Chiang, Z. Zilic, K. Radecka and J.S. Chenard, "Architectures of Increased Availability Wireless Sensor Network Nodes", Proceedings of ITC International Test Conference, pp. 1232-1244, 2004.

[10] Frey Hannes and Gorgen Daniel, "Geographical Clusterbased Routing in Sensing-Covered Networks", IEEE Transactions on Parallel and Distributed Systems, Vol. 17, No. 9, pp. 899-911, 2006.

[11] C. Karlof and D. Wagner, "Secure Routing in Wireless Sensor Networks: Attacks and Counter Measures", Proceedings of $1^{\text {st }}$ IEEE International Workshop on Sensor Network Protocols and Applications, pp. 293-315, 2003.

[12] W.K. Ma, B.N. Vo, S.S. Singh and A. Baddeley, "Tracking an Unknown Time-Varying Number of Speakers using TDOA Measurements: A Random Finite Set Approach", IEEE Transactions on Signal Processing, Vol. 54, No. 9, pp. 3291-3304, 2006.

[13] E.H. Callaway, "Wireless Sensor Networks Architectures and Protocols", Auerbach Publications, 2004.

[14] S. Hariri and H. Mutlu, "Hierarchical Modeling of Availability in Distributed Systems", IEEE Transactions on Software Engineering, Vol. 21, No. 1, pp. 50-56, 1995.

[15] M. Younis K. Akkaya, "A Survey on Routing Protocols for Wireless Sensor Networks", Ad Hoc Networks, Vol. 3, No. 3, pp. 325-349, 2005.

[16] H.O. Tan and I. Korpeoglu, "Power Efficient Data Gathering and Aggregation in Wireless Sensor Networks", SIGMOD Record, Vol. 32, No. 4, pp. 66-71, 2003.

[17] R.C. Shah and J. Rabaey, "Energy Aware Routing for Low Energy Ad Hoc Sensor Networks", Proceedings of IEEE International Conference on Wireless Communications and Networking, pp. 17-21, 2002.

[18] Y. Yu, D. Estrin and R. Govindan, "Geographical and Energy-Aware Routing: A Recursive Data Dissemination 
Protocol for Wireless Sensor Networks", Technical Report, Department Computer Science, University of California, 2001.

[19] A.T. Parameswaran et al., "Is RSSI a Reliable Parameter in Sensor Localization Algorithms: An Experimental Study", Proceedings of Workshop on Field Failure Data Analysis, pp. 1-5, 2009.
[20] D. Bein, V. Jolly, B. Kumar and S. Latifi, "Reliability Modeling in Wireless Sensor Networks", Proceedings of IEEE International Conference on Long Island Systems, Applications and Technology, pp. 1-8, 2005.

[21] R. Iyer and L. Kleinrock, "QoS Control for Sensor Networks", Proceedings of IEEE International Conference on Communications, pp. 517-521, 2003. 